\title{
Programa de estimulación intrauterina para mejorar el desarrollo bio - psico - social
}

\author{
Intrauterine stimulation program to improve bio - psycho - social development \\ Programa de estimulação intrauterina para melhorar o desenvolvimento \\ biopsicosocial
}

\section{Osmar Edson Sánchez Cueto}

osmaritowuawo@gmail.com

https://orcid.org/0000-0002-0615-6589

Recibido: noviembre 2019 Revisado: noviembre 2019

Universidad Privada Domingo Savio, Potosí, Bolivia

Aceptado: diciembre 2019

Publicado: enero 2020

\section{RESUMEN}

El presente artículo reconoce la importancia de la estimulación intrauterina o prenatal para contribuir con el desarrollo bio - psico - social del feto, del neonato y de la madre gestante. El objetivo de la investigación diseñar un programa de estimulación intrauterina que permita mejorar el desarrollo bio psico social del niño y de la madre gestante que acude a consulta en el centro de salud "San Pedro" en la ciudad de Potosí en Bolivia. Es una investigación proyectiva con un diseño de campo. La población estuvo constituida por 20 madres embarazadas y el personal de salud que las atiende en el centro de salud "San Pedro" en Potosí, Bolivia. Las técnicas para la recolección de los datos del diagnóstico fueron la encuesta y la entrevista y los instrumentos el cuestionario, la guía de entrevista y el grupo focal. La propuesta quedó constituida por talleres que permiten el desarrollo de competencias en estimulación intrauterina en sus dimensiones auditiva, visual y táctil o física.

Palabras clave: Estimulación intrauterina; desarrollo fetal; estimulación prenatal; programa
ABSTRACT

This article recognizes the importance of intrauterine or prenatal stimulation to contribute to the bio-psychosocial development of the fetus, the neonate and the pregnant mother. The objective of the research is to design an intrauterine stimulation program that allows improving the biopsycho-social development of the child and of the pregnant mother who comes to the "San Pedro" health center in the city of Potosi in Bolivia. The population consisted of 20 pregnant mothers and the health personnel who attend to them at the "San Pedro" health center in Potosí, Bolivia. The techniques for collecting the diagnostic data were the survey and the interview and the instruments the questionnaire, the interview guide and the focus group. The proposal was made up of workshops that allow the development of skills in intrauterine stimulation in its auditory, visual and tactile or physical dimensions.

Key words:

Intrauterine stimulation; fetal development; prenatal stimulation; program
RESUMO

Este artigo reconhece a importância da estimulação intrauterina ou pré-natal para contribuir com o desenvolvimento biopsicossocial do feto, do recém-nascido e da gestante. O objetivo da pesquisa é desenhar um programa de estimulação intrauterina que permita melhorar o desenvolvimento biopsicossocial da criança e da gestante que chega ao posto de saúde "San Pedro" na cidade de Potosí na Bolívia. É uma investigação projetiva com desenho de campo. A população é formada por 20 gestantes e os profissionais de saúde que as atendem no centro de saúde "San Pedro" em Potosí, Bolívia. As técnicas de recolha dos dados diagnósticos foram o inquérito e a entrevista e os instrumentos o questionário, o roteiro de entrevista e o grupo focal. A proposta foi composta por oficinas que permitem o desenvolvimento de habilidades em estimulação intrauterina em suas dimensões auditiva, visual e tátil ou física.

Palavras-chave:

Estimulação intrauterina; desenvolvimento fetal; estimulação pré-natal; programa 


\section{INTRODUCCIÓN}

La estimulación intrauterina es el resultado de numerosas investigaciones realizadas en Europa, Estados Unidos y América del Sur. Estos estudios generaron el sustento teórico para dar origen a la Psicología pre y perinatal. Autores como Verny y John (2009), Doan y Zimerman, (2003) y Caraballo (2016) coinciden en los efectos positivos de la estimulación intrauterina en la vida del niño, entre otras mencionan: evolución del desarrollo psicomotor, maduración del sistema nervioso, equilibrio socio emocional, relación neuronal con los órganos de los sentidos, vínculo materno-fetal.

En este mismo orden de ideas Pozo (2014) reporta que los bebes estimulados al nacer presentan mayor capacidad de seguimiento visual o auditivo ante un estímulo animado o inanimado, mejor control cefálico, movimiento y tono muscular y una más eficiente utilización de estrategias fisiológicas y posturales para mantener el equilibrio emocional al segundo día de nacimiento.

Los programas de estimulación intrauterina deben contemplar actividades para la vista, el oído y el movimiento; así como también, la transmisión de mensajes positivos y de aceptación hacia el feto en formación. El éxito de la aplicación de las propuestas depende de la frecuencia o repetición del estímulo, la intensidad con la cual se suministre el estímulo y su duración o tiempo de aplicación.

Otro elemento que se debe considerar para que sea exitosa la estimulación intrauterina es el conocimiento acerca del desarrollo del feto, al respecto Plevak, Schelotto, Bonifacino, Mussetti (2012) y Arteaga y García (2017) refieren que el sentido del tacto se desarrolla antes de la octava semana de gestación; en el séptimo mes se completa la formación del nervio acústico, pero desde el cuarto mes puede captar sonidos provenientes de su medio interno y del medio exterior y también puede sentir los cambios de posición y movimiento. El nervio óptico se forma en la séptima semana de gestación, pero los párpados se abren en la semana 27, pero el feto puede captar la luz aun con los párpados cerrados. A través de los neuroquímicos en la sangre de la madre el feto puede sentir el estrés o las emociones que ella esté viviendo, por esta razón es importante que la madre gestante realice ejercicios de relajación.

Vizcaíno (s.f.) concluye que a los tres meses de vida embrionaria, los órganos de los sentidos y los correspondientes centros cerebrales ya están formados. $Y$ durante los 6 meses siguientes estos órganos y centros se desarrollan y se especializan, según la calidad y la intensidad de los estímulos recibidos y la naturaleza de su función.

Por tanto cabe proponer que las prácticas de atención temprana se extiendan al periodo prenatal como estrategia de prevención, repercutiendo favorablemente en la salud materno-infantil.

La estimulación temprana es el conjunto de medios, técnicas, y actividades con base científica y aplicada en forma sistémica y secuencial que se emplea en los niños desde antes de nacer, con el objetivo de desarrollar al máximo sus capacidades cognitivas, físicas y psíquicas, permite también, evitar problemas os en el desarrollo y contribuir con los padres con eficacia y autonomía, en el cuidado y desarrollo del infante (Terre, 2000).

Corominas (2006) agrega que la estimulación intrauterina utiliza diferentes estímulos como son el sonido, música, voz materna, luz, vibración, presión y sabor para estimular los diferentes sentidos del bebé, de esta forma se favorece al desarrollo sensorial, mental y físico del niño.

La práctica profesional realizada en el centro de salud “San Pedro" permitió evidenciar la carencia de estimulación intrauterina al feto por parte de las madres gestantes, por esta razón se planteó la investigación para describir en primer lugar la 
estimulación intrauterina y con los resultados del diagnóstico diseñar un programa de estimulación temprana para mejorar el desarrollo bio psico social del feto y futuro neonato.

La interrogante de investigación que se consideró fue: ¿Cuáles son los elementos de un programa de estimulación intrauterina que permita mejorar el desarrollo bio psico social del niño y de la madre gestante que acude a consulta en el centro de salud "San Pedro" en la ciudad de Potosí en Bolivia? Y el objetivo guía de la investigación fue: Diseñar un programa de estimulación intrauterina que permita mejorar el desarrollo bio psico social del niño y de la madre gestante que acude a consulta en el centro de salud "San Pedro" en la ciudad de Potosí en Bolivia.

\section{MÉTODO}

Investigación proyectiva, cuyo objetivo fue diseñar un programa de estimulación para atender las carencias diagnosticadas. El enfoque es mixto porque se obtienen y procesan datos de forma cualitativa y cuantitativa, el diseño es de campo, pues los datos se recolectaron en el contexto natural.

El contexto en el cual se desarrolló la investigación es el centro de salud "San Pedro" ubicado en la ciudad de Potosí, en Bolivia. Este centro atiende a sus asegurados ofreciendo servicios en salud pública. Uno de los servicios que presta es la atención a mujeres en estado de gestación, otro servicio es el desarrollo infantil temprano dirigido a niños de 0 a 4 años.

La población del estudio fueron 20 madres gestantes que acuden al centro de salud "San Pedro" para su control prenatal y reciben como pago el bono "Juana Azurduy", el pago de este bono asegura la asistencia de forma regular al centro, ya que si no asisten no pueden obtener este beneficio.
Estas mujeres provienen de las áreas periféricas de la ciudad, como también son pacientes que han migrado de diferentes provincias rurales del departamento, lo que hace que muchas veces estén constantemente viajando a sus lugares de origen y no consigan asistir a sus controles prenatales y otros cuidados que se deben brindar durante esta etapa.

Las madres gestantes tienen edades comprendidas entre 15 y 35 años de edad, en su mayoría de ellas son bilingües porque utilizan la lengua materna que es el castellano como también el quechua, tienen entre 2 y 3 hijos mayores al que esta pronto a nacer, el grado de instrucción al cual llegó la mayoría de esta población es la culminación del nivel primario, existiendo excepciones que continúan con sus estudios en niveles superiores.

La mayoría de esta población aún trabaja durante su embarazo para ayudar económicamente a su familia, ya que provienen de familias numerosas y con niveles socioeconómicos limitados; por este motivo se sienten obligadas a trabajar y el trabajo que realizan muchas veces requieren de mucho esfuerzo físico.

Como muestra referencial también se consideró el personal de salud que atiende a las mujeres gestantes.

Las técnicas para la recolección de los datos fueron: la encuesta y la entrevista y los instrumentos estuvieron constituidos por: (a) el cuestionario estructurado dirigido a las madres, conformado por 8 ítems para medir las tres dimensiones de la variable; (b) la entrevista, dirigida al personal de salud, con 10 preguntas estructuradas acerca de su percepción sobre la estimulación intrauterina que realizan las madres gestantes; (c) El grupo focal o entrevista grupal se realizó con un grupo de 10 madres gestantes, quienes pudieron expresarse en relación a la estimulación intrauterina, con base en cuatro preguntas generadoras. Esto se realizó con el 
propósito de enriquecer la información del estado actual de las madres en estado de gestación, el instrumento consta de cuatro preguntas generales, que fueron estructuradas con base en la teoría consultada para su aplicación y están relacionadas con las tres dimensiones: estímulos auditivos, estímulos físicos y estímulos visuales.
La variable dependiente de la investigación es la estimulación intrauterina y su operacionalización con tres dimensiones: auditiva, visual y táctil o física se observa en el Cuadro 1.

Cuadro 1. Operacionalización de la variable.

\begin{tabular}{|c|c|c|c|c|c|}
\hline \multirow[t]{2}{*}{ Definición operativa } & \multirow[t]{2}{*}{ Dimensiones } & \multirow[t]{2}{*}{ Indicadores } & \multicolumn{3}{|c|}{ Instrumento } \\
\hline & & & Cuestionario & Entrevista & Grupo focal \\
\hline $\begin{array}{l}\text { La estimulación } \\
\text { intrauterina es un }\end{array}$ & $\begin{array}{l}\text { Estímulos } \\
\text { Auditivos }\end{array}$ & $\begin{array}{l}\text { Saluda a su bebé por las } \\
\text { mañanas }\end{array}$ & $x$ & $x$ & $\mathrm{x}$ \\
\hline $\begin{array}{l}\text { actividades que se } \\
\text { realiza durante la }\end{array}$ & & $\begin{array}{l}\text { Le habla durante el día } \\
\text { al bebé }\end{array}$ & $x$ & $x$ & $x$ \\
\hline $\begin{array}{l}\text { gestación, con el } \\
\text { objetivo de } \\
\text { desarrollar }\end{array}$ & & Le canta a su bebé & $\mathrm{x}$ & $\mathrm{x}$ & $\mathrm{x}$ \\
\hline $\begin{array}{l}\text { en los bebés sus } \\
\text { capacidades } \\
\text { cognitiva, física, }\end{array}$ & & $\begin{array}{l}\text { Escucha música con el } \\
\text { bebé }\end{array}$ & $x$ & $\mathrm{x}$ & $\mathrm{x}$ \\
\hline $\begin{array}{l}\text { emocional y } \\
\text { social. Las } \\
\text { prácticas } \\
\text { estimulatorias }\end{array}$ & & $\begin{array}{l}\text { Toca su abdomen con } \\
\text { sus manos en forma de } \\
\text { masajes }\end{array}$ & $x$ & $\mathrm{x}$ & $\mathrm{x}$ \\
\hline $\begin{array}{l}\text { consisten en la } \\
\text { aplicación } \\
\text { de estímulos }\end{array}$ & Estímulos Táctil & $\begin{array}{l}\text { Realiza masajes con } \\
\text { texturas en su abdomen }\end{array}$ & $x$ & $x$ & $x$ \\
\hline $\begin{array}{l}\text { de tipo auditivo, } \\
\text { táctil y visual }\end{array}$ & & Baila & $x$ & $x$ & $\mathrm{x}$ \\
\hline $\begin{array}{l}\text { los cuales } \\
\text { pueden aplicarse } \\
\text { en la etapa de la }\end{array}$ & & $\begin{array}{l}\text { Realiza ejercicios en el } \\
\text { agua }\end{array}$ & $x$ & $x$ & $\mathrm{x}$ \\
\hline $\begin{array}{l}\text { que los sentidos van } \\
\text { alcanzando }\end{array}$ & Estímulos Visuales & $\begin{array}{l}\text { Expone algún tipo de luz } \\
\text { artificial en el vientre }\end{array}$ & $x$ & $\mathrm{x}$ & $\mathrm{x}$ \\
\hline & & Expone al sol su vientre & $\mathrm{x}$ & $x$ & $x$ \\
\hline
\end{tabular}




\section{RESULTADOS}

Las derivaciones se presentan de acuerdo a los instrumentos.

\section{Guía de entrevista al personal de salud}

El personal de salud que atiende a las madres gestantes que acuden a la consulta en el centro "San Pedro" en la ciudad de Potosí, está de acuerdo, en su totalidad, que no existen conductas observables que ellos puedan mencionar si las madres están realizando estimulación intrauterina para cualquiera de las áreas visual, auditiva y táctil o física.

Agregan que la mayoría de estas "pacientes embarazadas" consideran el embarazo como una enfermedad, ya que tienen muchas creencias que durante el embarazo no se debe realizar ningún tipo de actividad porque afectaría al bebé.

Es de hacer notar que el personal de salud se refiere a las madres gestantes como "pacientes embarazadas", esto es un indicador que también consideran el embarazo como un tipo de enfermedad, muy similar como lo consideran a su juicio las mujeres embarazadas.

Todo el personal de salud está de acuerdo que es importante la incorporación de un programa en estimulación intrauterina porque esto trae beneficios en las relaciones entre el bebé y su familia, en la condición física y emocional de la madre gestante y en el desarrollo del feto.

\section{Resultados del cuestionario aplicado a las madres gestantes}

Este cuestionario brinda información acerca de la estimulación intrauterina en sus tres dimensiones: visual, auditiva, táctil

\section{Dimensión auditiva}

La dimensión auditiva capta los estímulos de sonido que la madre gestante realiza para comunicarse con su hijo. Los resultados se resumen en el Gráfico 1.

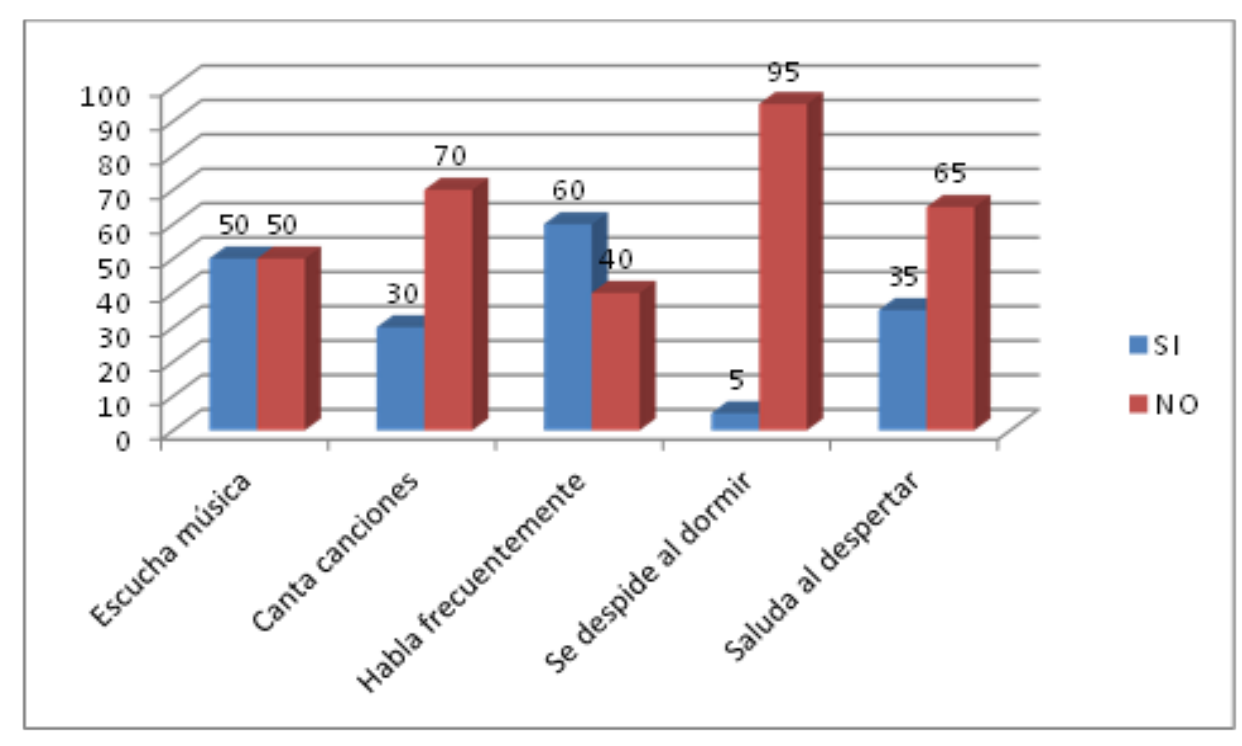

Gráfico 1. Dimensión auditiva. 
Se observa que el $60 \%$ de las madres le hablan con frecuencia al feto, el $50 \%$ cuando escucha música involucra a su bebé en la escucha. Los otros indicadores cantarle canciones, despedirse al dormir y saludar al despertar tienen resultados más negativos $70 \%$, $95 \%$ y $65 \%$ respectivamente no consideran estas conductas.

Estos datos indican que las madres gestantes no realizan estimulaciones auditivas a sus bebés, como el hablarle desde el saludo a sus hijos, el cantarles, el escuchar música. En contraste con lo que se menciona en el marco teórico, que estas actividades si estimulan al sentido del oído de los bebés aún no nacidos.

\section{Dimensión táctil o de estímulos físicos}

Esta dimensión capta las conductas que hacen referencia a la comunicación a través del movimiento que puede ejecutar la madre gestante involucrando al feto en su desarrollo sensorial, los resultados en el Gráfico 2.

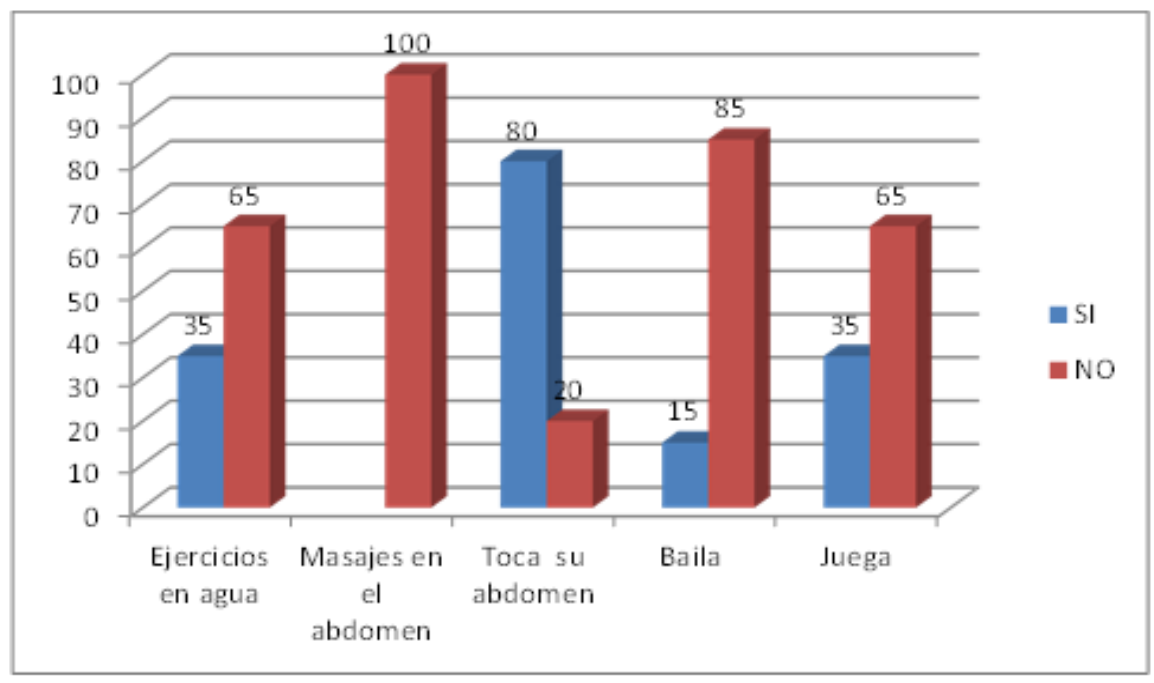

Gráfico 2. Dimensión táctil estímulos físicos.

El $80 \%$ de las madres gestantes tocan con frecuencia su abdomen, los otros indicadores son negativos solo el $35 \%$ realiza ejercicios en agua y juegos con su bebe; el $85 \%$ no baila.

Las madres gestantes respondieron que nunca realizaron este tipo de actividades como parte de la estimulación, porque desconocían su importancia, además que desde el momento que se enteran de su embarazo, su ansiedad crece lo que imposibilitaría a realizar actividades de movimiento como el baile.

También mencionan que los juegos son para niños ya nacidos niños grandes, indicando que el bebé aún en vientre, no sabe sobre juegos porque no siente, lo que contrariamente se demuestra en el marco teórico de la investigación, que el bebé sí percibe estímulos externos y es necesario para su desarrollo sensorial táctil.

Estos datos indican que no se realiza la estimulación táctil por parte de las madres gestantes en el área motriz, como el hacer masajes con distintas texturas o realizar actividades que produzcan movimiento en el cuerpo de la madre gestante y generar estimulación al bebé.

\section{Dimensión visual}

Esta dimensión capta la exposición a la luz de forma consciente que se realiza en el abdomen para estimular la visión del feto. (Ver Gráfico 3). 


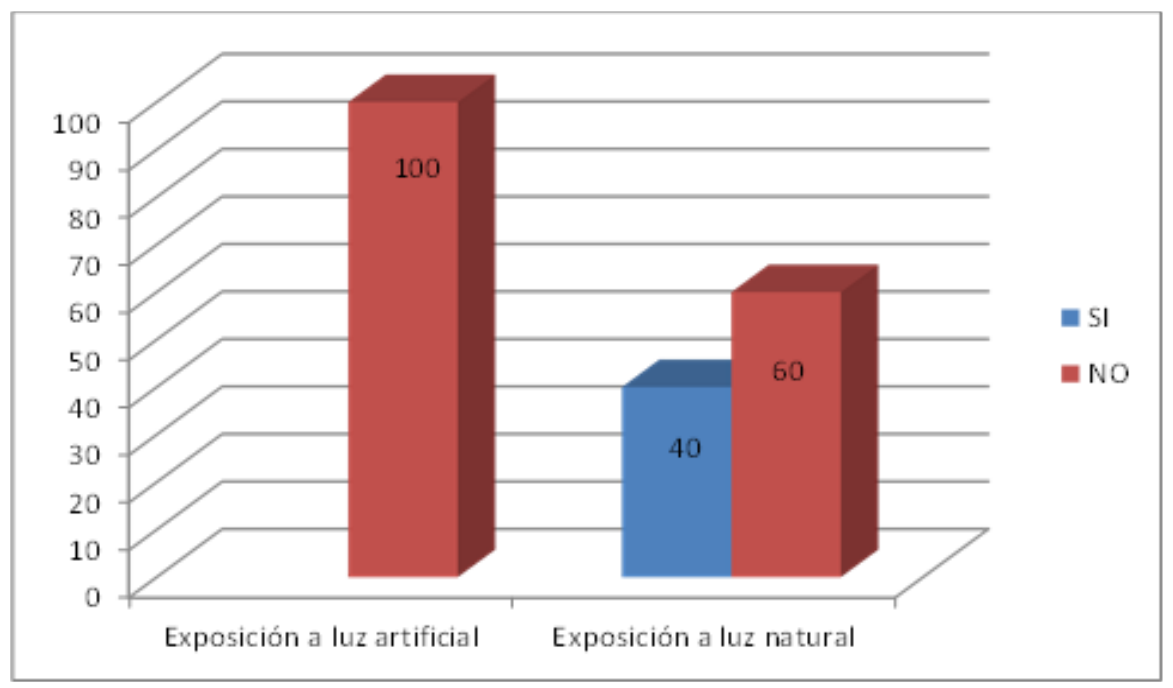

Gráfico 3. Dimensión visual.

Ninguna de las madres gestantes utiliza luz artificial en su vientre, $40 \%$ señalan que si exponen su vientre al sol, el $60 \%$ de la población gestante no realiza esta actividad, mencionan que la luz del sol puede ser dañina para ellas y para sus bebés en vientre, ya que muchas veces sintieron sofocación cuando se expusieron al sol, otra razón que exponen es el exceso de actividades que tienen diariamente como el trabajo les impide hacerlo.

Estos datos indican que no hay carencias en la estimulación visual del feto.

\section{Resultados del Grupo focal}

El grupo focal se realizó con 10 madres gestantes, con este instrumento las madres pudieron expresar sus opiniones con respecto a la estimulación intrauterina.

La opinión de la mayoría es que no realizan ningún tipo de estimulación o no lo hacen de forma consciente y frecuente porque no conocen la importancia de esta estimulación en el desarrollo del bebe. Consideran que no tienen tiempo para ello porque tienen más hijos que atender y adicionalmente porque trabajan varias horas al día. También exponen el temor de hacerle daño al feto con alguna de estas acciones.
Algunos de los factores que están interviniendo de forma negativa e impiden la estimulación intrauterina son la falta de conocimiento acerca de los beneficios de la estimulación, la falta de tiempo y las creencias acerca de la condición del embarazo como una limitación.

Con base en estos resultados se diseña el programa de estimulación intrauterina dirigido a las madres gestantes de este centro de salud.

A continuación se presenta de forma parcial el programa.

\section{PROPUESTA}

\section{Programa de estimulación intrauterina}

\section{Taller 1. Socialización de la propuesta}

Este taller está dirigido al personal que atiende mujeres gestantes que acuden del centro de salud centro "San Pedro" de la ciudad de Potosí. El taller tiene la duración de 2 horas y 15 minutos, tiene como objetivo informar acerca de los alcances y beneficios de la propuesta de estimulación intrauterina. (Véase Cuadro 2). 
Cuadro 2. Taller 1.

Nombre del taller: Socialización de la propuesta de orientación para la promoción de la estimulación intrauterina. Participantes: El personal del centro de salud

Objetivo: Explicar los alcances de la propuestas estimulación intrauterina

\begin{tabular}{llcl}
\hline \multicolumn{1}{c}{ Etapa } & \multicolumn{1}{c}{ Actividades - temáticas } & Tiempo & \multicolumn{1}{c}{ Materiales } \\
\hline Inicio & $\begin{array}{l}\text { Se realizará la bienvenida, con una dinámica llamada mi plato } \\
\text { favorito, la cual trata de que cada participante mencione su plato } \\
\text { preferido, luego mencione su nombre por delante }\end{array}$ & $15^{\prime}$ & $\begin{array}{l}\text { Velas } \\
\text { Cartulina, } \\
\text { Marcadores }\end{array}$ \\
Desarrollo & $\begin{array}{l}\text { Se dará comienzo a la explicación sobre las características de la } \\
\text { estimulación intrauterina y su importancia, presentando a detalle } \\
\text { sus beneficios y el cómo se aplicará la propuesta }\end{array}$ & $1 \mathrm{~h}: 30^{\prime}$ & $\begin{array}{l}\text { Reproductor de data } \\
\text { desplaye, videos de } \\
\text { apoyo, música de } \\
\text { apoyo }\end{array}$ \\
Final & $\begin{array}{l}\text { Se realizará una retroalimentación con evaluación general. } \\
\text { Agradecimiento al personal }\end{array}$ & $30^{\prime}$ & Refrigerio \\
\hline
\end{tabular}

\section{Talleres para las madres gestantes}

Estos talleres son cinco, se realizarán una vez al mes con las madres gestantes que tienen el beneficio del servicio bono "Juana Azurduy" en el centro de salud "San Pedro".

Cuadro 3. Taller 2. Socialización de la propuesta para las madres.

Nombre del taller: Socialización del programa de orientación de estimulación intrauterina

Participantes: Madres gestantes

Objetivo: Informar los beneficios y características de la estimulación intrauterina

\begin{tabular}{llcl}
\hline \multicolumn{1}{c|}{ Etapa } & \multicolumn{1}{c}{ Actividades - temáticas } & Tiempo & Materiales \\
\hline Inicio & Bienvenida con una dinámica llamada “ la mímica de nombres” & $15^{\prime}$ & \\
Desarrollo & $\begin{array}{l}\text { Se explicarán las características, la importancia, y los beneficios } \\
\text { de la estimulación intrauterina. }\end{array}$ & $1 \mathrm{~h}: 30^{\prime}$ & $\begin{array}{l}\text { Reproductor de data } \\
\text { desplaye, videos de } \\
\text { apoyo, música de } \\
\text { apoyo }\end{array}$ \\
Final & $\begin{array}{l}\text { Se realizará una retroalimentación, para luego realizar una } \\
\text { evaluación general. Agradecimiento e invitación al refrigerio. }\end{array}$ & $30^{\prime}$ & Refrigerio \\
\hline
\end{tabular}


Cuadro 4. Taller 3. Estimulación auditiva.

Nombre del taller: Estimulación auditiva

Participantes: Madres gestantes

Objetivo general: Desarrollar competencias cognitivas y prácticas de la estimulación auditiva intrauterina

\begin{tabular}{llcl}
\hline \multicolumn{1}{c}{ Etapa } & \multicolumn{1}{c}{ Actividades - temáticas } & Tiempo & \multicolumn{1}{c}{ Materiales } \\
\hline Inicio & $\begin{array}{l}\text { Se realizará la bienvenida con una dinámica } \\
\text { llamada “miembros de la familia” }\end{array}$ & $15^{\prime}$ & $\begin{array}{l}\text { Cartulina } \\
\text { Marcadores }\end{array}$ \\
Desarrollo & $\begin{array}{l}\text { Exposición y discusión de la estimulación auditiva, } \\
\text { su importancia y sus beneficios. }\end{array}$ & $45^{\prime}$ & $\begin{array}{l}\text { Reproductor de data desplaye, } \\
\text { videos de apoyo, música } \\
\text { de apoyo }\end{array}$ \\
Final & $\begin{array}{l}\text { Ejercicios de estimulación auditiva con la participa- } \\
\text { ción activa de las madres gestante. }\end{array}$ & $1 \mathrm{~h}$ & $\begin{array}{l}\text { Colchonetas, reproductor de } \\
\text { audio y video, sonajeros, botellas } \\
\text { rellenas de arroz, piedras. } \\
\text { instrumentos musicales, Embudo, } \\
\text { manguera }\end{array}$
\end{tabular}

Cuadro 5. Taller 4. Estimulación táctil.

Nombre del taller: Estimulación táctil o física

Participantes: Madres gestantes

Objetivo: Desarrollar competencias cognitivas y prácticas de la estimulación auditiva intrauterina

\begin{tabular}{llcl}
\hline \multicolumn{1}{c}{ Etapa } & \multicolumn{1}{c}{ Actividades - temáticas } & Tiempo & \multicolumn{1}{c}{ Materiales } \\
\hline Inicio & Bienvenida con la dinámica “ masaje grupal” & $15^{\prime}$ & \\
Desarrollo & $\begin{array}{l}\text { Se procederá a explicar sobre la estimulación táctil, } \\
\text { su importancia y sus beneficios. }\end{array}$ & $45^{\prime}$ & $\begin{array}{l}\text { Reproductor de } \\
\text { data desplaye, videos de apoyo }\end{array}$ \\
Final & $\begin{array}{l}\text { Ejercicios de estimulación táctil con la participa- } \\
\text { ción activa de las madres gestantes. }\end{array}$ & $1 \mathrm{~h}$ & $\begin{array}{l}\text { - Colchonetas } \\
\text { - Esponja para lavar platos } \\
\text { Pelotas de tamaño grande, } \\
\text { mediano y pequeño }\end{array}$ \\
& & & $\begin{array}{l}\text { - Pedazos de tela, con textura } \\
\text { suave y áspera }\end{array}$ \\
\hline
\end{tabular}


Cuadro 6. Taller 5. Estimulación visual.

Nombre del taller: Estimulación visual

Participantes: Madres en estado gestación del centro de salud San Pedro

Objetivo: Desarrollar competencias cognitivas y prácticas de la estimulación visual intrauterina

\begin{tabular}{llcl}
\hline Etapa & \multicolumn{1}{c}{ Actividades - temáticas } & Tiempo & \multicolumn{1}{c}{ Materiales } \\
\hline Inicio & $\begin{array}{l}\text { Se realizará una dinámica denominada " pareja a } \\
\text { ciegas" }\end{array}$ & $20^{\prime}$ & $\begin{array}{l}\text { - Cajas de cartón } \\
\text { - Venda oscura }\end{array}$ \\
Desarrollo & $\begin{array}{l}\text { Se explicará sobre la estimulación táctil, su } \\
\text { importancia y sus beneficios }\end{array}$ & $45^{\prime}$ & $\begin{array}{l}\text { Reproductor de data } \\
\text { desplaye, videos de apoyo }\end{array}$ \\
Final & $\begin{array}{l}\text { Ejercicios, actividades de estimulación visual con la } \\
\text { participación activa de las madres gestantes (Ane- } \\
\text { xo } 4 \mathrm{~g})\end{array}$ & $1 \mathrm{~h}$ & $\begin{array}{l}\text { - Colchonetas } \\
\text { - Linternas }\end{array}$ \\
& & & $\begin{array}{l}\text { - Papel celofán de colores } \\
\text { - Pedazo de tela }\end{array}$ \\
\hline
\end{tabular}

Cuadro. Taller 6. Estimulación motora.

Nombre del taller: Estimulación motora

Participantes: Madres en estado gestación del centro de salud San Pedro

Objetivo: Desarrollar competencias cognitivas y prácticas de la estimulación motora intrauterina

\begin{tabular}{llcl}
\hline \multicolumn{1}{c}{ Etapa } & \multicolumn{1}{c}{ Actividades - temáticas } & Tiempo & \multicolumn{1}{c}{ Materiales } \\
\hline Inicio & $\begin{array}{l}\text { Se realizará una dinámica denominada “Un grupo } \\
\text { balanceado" }\end{array}$ & $15^{\prime}$ & \\
Desarrollo & $\begin{array}{l}\text { Se explicará sobre la } \\
\text { estimulación motora, tanto su importancia y sus } \\
\text { beneficios. }\end{array}$ & $45^{\prime}$ & $\begin{array}{l}\text { Reproductor de data } \\
\text { desplaye, videos de apoyo }\end{array}$ \\
Final & $\begin{array}{l}\text { Ejercicios, actividades de estimulación motora con } \\
\text { la participación activa de las madres gestantes. }\end{array}$ & $1 \mathrm{~h}$ & $\begin{array}{l}\text { - Colchonetas } \\
\text { - Papel celofán de colores } \\
\text { - Pedazo de tela }\end{array}$ \\
\hline
\end{tabular}




\section{Evaluación de la aplicación del programa}

Conductas durante la ejecución del programa de estimulación intrauterina. El estado físico y emocional de la madre gestante una vez realizada la actividad de la estimulación. El interés que muestre la población de madres gestante por asistir a las sesiones y talleres de estimulación intrauterina.

\section{CONCLUSIONES}

Las madres gestantes de la muestra no poseen información acerca de la estimulación intrauterina y desconocen las ventajas. También desconocen los distintos tipos de estimulación visual, auditiva y física.

Para estimular al niño en su etapa prenatal la madre gestante debe tener conocimiento de las técnicas, el procedimiento de aplicación.

Las madres gestantes que conocen las técnicas de estimulación intrauterina se sienten interesadas en realizar las actividades para mejorar el desarrollo de su hijo por nacer.

El interés y la motivación de las madres a participar en un programa de estimulación intrauterina van en aumento a medida que obtienen mayor información acerca de las ventajas en el desarrollo motor, psicológico y social en los niños.

\section{REFERENCIAS}

Arteaga, M. y García, M. (2017). Embriología humana y biología del desarrollo. ( $2^{a}$.ed.) México: Panamericana
Brand G. (2003). Estimulación prenatal. El inicio de una educación ecológica del neocórtex. Pediatría al Día, 19(5):50-54

Caraballo A. (2006). Cómo estimular al bebé desde el embarazo. Editora de Guialnfantil. com. https://www.guiainfantil.com/articulos/ embarazo/como-estimular-al-bebe-desde-elembarazo/

Corominas, C. (2006). Embarazo y primer año de vida. Madrid: Palabra S.A

Doan, H. M. y Zimerman, A. (2003). Conceptualizing prenatal attachment: Toward a multidimensional view. Journal of Prenatal and Perinatal Psychology and Health, 18, p. 109

Plevak A., Schelotto M., Bonifacino N. y Mussetti D. (2012). Consulta pediátrica en la primera infancia: una oportunidad para la detección de indicadores de riesgo en el desarrollo emocional. Experiencia de tamizaje e intervención temprana. Arch. Pediatr. Urug. [Revista en línea] 83(2), pp. 80-86. http://www.scielo.edu. uy/scielo.php?script=sci_arttext\&pid=S1688$12492012000200002 \& \operatorname{lng}=\mathrm{es}$

Pozo, M. (2014). Pediatría Integral. Valencia: MIR de la SEPEAP

Terre, O. (2000). Criterios y visión de la estimulación infantil. Bogotá: Alianza

Verny, T. y John, K. (2009). La vida secreta del niño antes de nacer. Horacio González (Trad.). España: Urano

Vizcaíno, P. (s.f.). La estimulación intrauterina [Ponencia en línea]. http://www.waece.org/ biblioteca/pdfs/d007.pdf 